\title{
Quality Risk Aversion, Conjectures, and New Product Diffusion
}

Francesco Bogliacino and Giorgio Rampa

\section{EERI Research Paper Series No 27/2009}

ISSN: 2031-4892

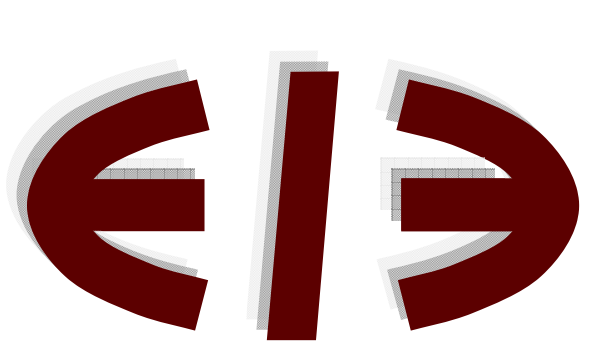

EERI

Economics and Econometrics Research Institute

Avenue de Beaulieu

1160 Brussels

Belgium

Tel: +3222993523

Fax: +322 2993523

www.eeri.eu

Copyright (C) 2009 by Francesco Bogliacino and Giorgio Rampa 


\title{
Quality Risk Aversion, Conjectures, and New Product Diffusion
}

\author{
Francesco Bogliacino* Giorgio Rampa ${ }^{\dagger}$
}

August 26, 2009

\begin{abstract}
In this paper we provide a generalization of the standard models of the diffusion of a new product. Consumers are heterogeneous and risk averse, and the firm is uncertain about the demand curve: both learn from past observations. The attitude towards risk has important effects with regard to the diffusion pattern.

In our model, downward-biased signals to consumers can prevent the success of the product, even if its objective quality is high: a "lockin" result. We show in addition that the standard logistic pattern can be derived from the model. Finally, we discuss the asymptotic behavior of the learning dynamics, with regard to the multiplicity and the stability of equilibria, and to their welfare properties.
\end{abstract}

Keywords: Heterogeneity, Multiple equilibria, Lock-in, Product diffusion, Risk aversion.

JEL Classification numbers: L15, D81, O33.

\footnotetext{
*EAFIT University, University of Genoa and University of Pavia, e-mail: francesco.bogliacino@gmail.com

${ }^{\dagger}$ Corresponding author, University of Genoa, e-mail: g.rampa@unige.it
} 


\section{Introduction}

Innovation diffusion has been studied mainly from two perspectives: the speed of diffusion, and the conditions favoring or hampering it ${ }^{1}$.

As to the former perspective, given the empirical $S$-shape of many diffusion processes, several papers assume $a b$ initio a logistic curve deemed to describe the diffusion path. The parameters of this curve determine the speed and the time of market saturation (Mahajan-Peterson 1978-1985, Dodson-Muller 1978, Gerosky 2000). The reason often presented for such a choice is that diffusion processes resemble epidemic phenomena, which are described by logistic functions (Hivner et al. 2003). One limitation of these studies is that they do not consider the motivations of agents, using aggregate models which boil down to single equations of information contagion. Due to the ability to fit data, these contagion models are the main ingredient of the management literature on product diffusion, where a generalization has been provided by the so-called Bass-like demand models (Bass 1969), which includes both internal and external (to the group) source of diffusion.

Among theories that try to give a more satisfactory description of the $m i$ cro process of diffusion, one can include the large strand of literature, more sociological in spirit, where some agent heterogeneity is assumed. In these models, however, the decision process is based mainly on different individual abilities to resist to some social effect: the "fundamentals" are, e.g., the individual propensities not to be displaced from average behavior ("bandwagon effect": Abrahamson-Rosenkopf, 1993 and 1999), or the proximity between agents, affecting the strength of contagion transmission (Cowan-Jonard 2003 and 2004), or some activation threshold, representing the ability of nodes to diffuse the contagion around themselves (Lopez Pintado-Watts, 2006).

The economics literature has been more interested in grounding the logistic diffusion pattern over a choice process more close in spirit to standard choice theory. The increasing-return models à la Arthur (1994), or more recently Mukoyama (2006), providing a micro-foundation for Rosenberg's learning by using, are examples of one of the roads followed; learning models in the context of bayesian equilibria in network games (e.g. Jackson-Yariv 2007) are examples of another one. Yet a different family of models is closer to traditional modeling of choice under uncertainty: here initial uncertainty is described in terms of agents' subjective probability distributions on the profitability of an innovation; distributions are updated in each period using Bayes formula and observing other agents' adoption choices (Jensen 1982,

\footnotetext{
${ }^{1}$ For an overall review of the literature, see Hall 2005.
} 
Feder-O'Mara 1982, Tsur et al. 1990, Chatterjee-Eliashberg 1990, Young 2006). The differences among subjective distributions generate different adoption times on the part of different agents: then the overall innovation diffusion is not instantaneous, coherently with traditional logistic models.

More recently Young (2007) summed up and characterize the above literature, proposing a model taxonomy in terms of inertia, contagion, conformity, social learning and moving equilibria; he concentrated on deriving the acceleration patterns implied by the different setups.

As regards the factors favoring or hampering diffusion, we have basically two approaches: either the destiny of the innovation is uniquely predetermined by some feature of the network (e.g. in contagion-type models), or it depends on some objective feature of the product: a "good" innovation will always spread out (Jensen 1982, Chatterjee-Eliashberg 1990).

To find models in which the destiny of an innovation is not uniquely predetermined, one must consider theories that incorporate some form of increasing returns: as a consequence, if the initial number of adopters fails to exceed a certain threshold, the new product is not successful even if it is a high-quality one ("lock-in" phenomena: Arthur, 1989 and 1994, Amable 1992, Agliardi 1998, Aoki-Yoshikawa 2002). In these models increasing returns are taken as an objective a-priori feature of the market.

The present paper couples bayesian behavior and agent heterogeneity: facing a new product consumers are uncertain about its quality, so they maintain subjective priors over it, updating them using available information. We concentrate on this kind of heterogeneity, and not on differences in fundamental parameters such as tastes and technologies. We distance ourself from previous literature in that (a) we explore the implications of risk-aversion, and (b) we look at both sides of the market, demand and supply. Although recognized by some authors (Tsur et al. $1990^{2}$, Verbrugge $2000^{3}$ ), the former aspect has usually been neglected. Risk aversion might imply that the consumers' willingness to pay increases independently of objective quality, if their subjective uncertainty declines in time: uncertainty reduction, in turn, comes from signals of previous adoptions on the part of

\footnotetext{
${ }^{2}$ Tsur et al. (1990), assuming risk aversion in the context of infinite horizon maximization, obtain the counterfactual result that more risk-averse firms are more inclined to adopt an innovation whose profitability is uncertain: in fact, in their setup, choosing a new technology allows diversification, which reduces future risk.

${ }^{3}$ Verbrugge (2000) starts from a utility which is straight-off decreasing in the variance of a prospect, and assumes that variance decreases objectively in time due to previous adoptions. In a sense, objective increasing returns are translated from mean to volatility.
} 
other consumers. This is a major endogenous positive-feedback mechanism ${ }^{4}$. Regarding the joint analysis of demand and supply: to our knowledge, a part from the case of Ho et al 2002 (who consider the role of capacity constraint in a Bass-like model) ours is the first organic attempt to address the problem and to examine equilibrium solutions. In our setup a single risk-neutral firm $^{5}$, not knowing the form of the market demand curve, maintains and updates a subjective conjecture about it.

The main contributions of the paper are the following. As regards lockin theory, we show that there is a positive probability that a high-quality innovation fails to diffuse, due to downward-biased signals ${ }^{6}$ and, above all, to consumers' "pessimism": the latter feature is incorporated in the second -not only first- moments of their priors, due to risk aversion. As regards market equilibria, we prove the existence of a continuum of conjectural equilibria, and characterize their welfare and stability properties. On both sides we provide analytical results instead of relying on simulation exercises, as is common in a large part of the literature.

The paper proceeds as follows: Section 2 sets up the model; Section 3 studies the possibility of lock-in phenomena and makes some arguments about the speed of diffusion; Section 4 defines conjectural equilibria and studies their properties; Section 5 concludes and offers some hints about possible future extensions. All proofs are confined in the Appendix.

\section{Setup}

\subsection{Quality}

Consider a new product launched by a firm at some initial date "0". There are $M$ potential buyers (consumers) of this product who can start buying the product from date 1 onward; time is discrete.

Each purchase of this product results in a stochastic quality signal $\lambda$ to a buyer, independently of the quantity acquired: randomness is due to, e.g., production and/or delivery factors. For the sake of simplicity, $\lambda$ is assumed

\footnotetext{
${ }^{4}$ This direction of analysis was anticipated in Rampa 2002.

${ }^{5}$ The introduction of a new good implies, as usual, monopoly. In addition, we follow the standard road of having risk-neutrality on the producer side.

${ }^{6}$ Besides being affine to other lock-in results, this is also close in spirit to the BanerjeeFudenberg (2004) model, where in presence of biased signals agents may converge to an inefficient outcome.
} 
to be a normal variable ${ }^{7}$ with true mean $\mu_{T}$ and precision $^{8} r$ :

$$
\lambda \sim N\left(\mu_{T}, r^{-1}\right)
$$

where " $"$ " means "is distributed as". Call $f_{T}(\lambda)$ the true distribution of $\lambda$.

The precision $r$ is assumed to be known to consumers, while the mean $\mu_{T}$ is not ${ }^{9}$. If consumer $i$ decides to buy a positive quantity of the product at date $t \geq 1$, she receives a quality signal thereof, say $\lambda_{i, t}$ : we assume that $\lambda_{i, t}$ is i.i.d. in time and among consumers, with distribution given by (2.1). This signal is publicly announced, so that each consumer can compute the sample average $\bar{\lambda}_{t}$ :

$$
\bar{\lambda}_{t}=\frac{1}{M_{t}} \sum_{i \in C_{t}} \lambda_{i, t}
$$

where $C_{t}$ is the set of consumers buying a positive quantity at date $t$, and $M_{t} \leq M$ is the cardinality of that set.

\subsection{Consumer's choice and learning}

For any given quality $\lambda$, consumer $i$ yields the following time-separable quasilinear utility from her consumption at date $t \geq 1$ :

$$
U\left(q_{i, t}, \lambda, m_{i, t}\right)=\left(q_{i, t}\right)^{\delta}\left(A-e^{-\lambda}\right)+m_{i, t}
$$

where $q_{i, t}$ is the quantity of the new product, and $m_{i, t}$ is that of a "money" (numeraire) good, consumed at that date; $0<\delta<1$, and $A>0$ are common to all consumers. This commonality assumption helps concentrating on a different source of heterogeneity, namely conjectures, as discussed below.

The utility function (2.3) has been chosen for analytical simplicity and, though somehow unusual, it has some convenient properties. First of all, besides utility being strictly concave in quantity, the marginal utility of quantity increases in quality. In fact one has $\partial^{2} U /(\partial q \partial \lambda)=A \delta q^{\delta-1} e^{-\lambda}>0$ (omitting subscripts for simplicity), meaning that the consumer wishes to purchase more if quality is higher, for given price.

Secondly, $i$ is "risk averse" with respect to quality, a property that is most important in the present setting. More precisely, not only total

\footnotetext{
${ }^{7}$ Normality implies that quality might be negative (and much so): the meaning of this is that the new product might be a "very nasty" one.

${ }^{8}$ Recall that a variable's precision is the inverse of its variance

${ }^{9}$ This assumption is made in order to keep things as simple as possible: if also the precision were unknown, one would need a bivariate distribution, instead of a univariate one, in expression (2.4) below.
} 
utility, but also marginal utility is concave in quality: $\partial^{3} U /\left(\partial q \partial \lambda^{2}\right)=$ $-A \delta q^{\delta-1} e^{-\lambda}<0$. This suggests that a higher variance of quality tends to depress (expected) marginal utility and hence consumption, for given price.

Consumers know that quality is normally distributed, and know its precision $r$, but they are uncertain about its true mean $\mu_{T}$. Here we assume heterogeneity among consumers. At date $t \geq 0$, consumer $i$ holds a personal conjecture over $\mu_{T}$, taking the form of a normal distribution:

$$
\mu_{T} \sim_{i, t} N\left(\mu_{i, t},\left(\tau_{i, t}\right)^{-1}\right)
$$

where " $\sim_{i, t}$ " means "is, according to $i$ at date $t$, distributed as", and the "hyper-parameters" $\mu_{i, t}$ and $\tau_{i, t}$ are, respectively, $i$ 's conjectured mean and precision at that date. Call $f_{i, t}(\mu)$ this personal conjectured distribution.

Let $y$ be consumer $i$ 's income ( $y$ is constant in time and equal among consumers), and call $p_{t}$ the price announced by the firm at date $t$. Then $i$ 's expected-utility maximization at date $t \geq 1$ requires to solve:

$$
\max _{q_{i, t}, m_{i, t}} E_{i, t-1}\left[q_{i, t}^{\delta}\left(A-e^{-\lambda}\right)+m_{i, t}\right] \quad \text { s.t. } \quad p_{t} q_{i, t}+m_{i, t}=y
$$

The expected value $E_{i, t-1}[\cdot]$ is taken w.r.t. the subjective conjecture $f_{i, t-1}(\mu)$, defined after expression (2.4). Notice the timing of events: in order to formulate her demand at date $t$, the consumer observes the price announced at that date, but her conjecture was formed at date $t-1$, before receiving any signal on quality at date $t$.

After routine calculation ${ }^{10}$, the solution to the above problem is

$$
q_{i, t}=p_{t}^{\frac{1}{(\delta-1)}}\left[\delta\left(A-e^{(2 r)^{-1}+\left(2 \tau_{i, t-1}\right)^{-1}-\mu_{i, t-1}}\right)\right]^{\frac{1}{(1-\delta)}}
$$

together with $m_{i, t}=y-p_{t} q_{i, t}$.

As expected the demand for the new product, besides decreasing in price with elasticity $\frac{1}{1-\delta}$, increases with expected quality $\mu_{i, t-1}$ and more importantly it increases with precision, i.e. decreases with variance. This is a key result, interpreting quality risk aversion on the part of consumers. Precision, in turn, splits into objective and subjective precision: of course the latter is more interesting from our present point of view.

\footnotetext{
${ }^{10}$ In particular, recall that if $\lambda$ is a normal variable with mean $\mu$ and precision $r$, then $e^{-\lambda}$ is lognormal with mean $e^{-\mu+1 / 2 r}$. It follows that, if $r$ is known and the prior over $\mu$ is normal with hyper-parameters $\mu_{i}$ and $\tau_{i}$, the subjectively expected value of $e^{-\lambda}$ is $e^{-\mu+1 / 2 r+1 / 2 \tau_{i}}$
} 
In (2.5) it must be intended that

$$
\text { if } \quad\left(A-e^{(2 r)^{-1}+\left(2 \tau_{i, t-1}\right)^{-1}-\mu_{i, t-1}}\right) \leq 0 \quad \text { then } \quad q_{i, t}=0
$$

meaning that the consumer sets her demand equal to zero, independently of price, if the product is judged "not worthy", i.e. if its subjectively expected quality is somehow too low.

In order to avoid trivial cases, namely a so "bad" objective quality distribution that nobody - even knowing it with certainty- would buy any quantity, we introduce the following

Assumption 1. $\left(A-e^{(2 r)^{-1}-\mu_{T}}\right)>0$

Of course, Assumption 1 leaves room for a null demand on the part of $i$ at some finite date $t$, depending on $f_{i, t-1}(\mu)$, i.e. on $\mu_{i, t-1}$ and on $\tau_{i, t-1}$.

According to a standard result in consumer theory ${ }^{11}$, the condition for having a positive demand for the money good is $\partial U / \partial q_{\mid q=y / p}<p$, that in our case can be written as

$$
y^{\frac{\delta-1}{\delta}}\left[\delta\left(A-e^{(2 r)^{-1}+\left(2 \tau_{i, t-1}\right)^{-1}-\mu_{i, t-1}}\right)\right]^{\frac{1}{\delta}}<p
$$

Since the eventuality that the consumer spends all her income on the new good adds only analytical complication, and no further insight, we assume that income is high enough to satisfy constraint (2.7).

Defining

$$
K_{i, t} \equiv\left[\delta\left(A-e^{(2 r)^{-1}+\left(2 \tau_{i, t-1}\right)^{-1}-\mu_{i, t-1}}\right)\right]^{\frac{1}{(1-\delta)}}
$$

one computes the market demand at date $t$ as follows:

$$
Q_{t}^{D}=p_{t}^{\frac{1}{(\delta-1)}} \sum_{i \in C_{t}} K_{i, t}
$$

where $C_{t}$ was defined in section 2.1 .

As regards consumers' learning, we follow standard Bayesian lines: $i$ 's conjecture (2.4) must be thought of as her posterior distribution at date $t^{12}$.

\footnotetext{
${ }^{11}$ Namely, a corner solution can be a consumer equilibrium only if, in the point where the expenditure on a single good exhausts income, the marginal rate of substitution is higher than the relative price.

${ }^{12}$ With our timing convention, $f_{i, 0}(\mu)$ indicates consumer's initial prior.
} 
The posterior at $t$ is computed recursively starting from the posterior at $t-1$, and observing the number of buyers $M_{t}$ and the quality sample average $\bar{\lambda}_{t}$ at date $t$ : the latter terms were defined in expression (2.2). Given our assumptions, it turns out ${ }^{13}$ that the updated hyper-parameters are:

$$
\mu_{i, t}=\frac{\tau_{i, t-1} \mu_{i, t-1}+r M_{t} \bar{\lambda}_{t}}{\tau_{i, t-1}+r M_{t}} \quad \text { and } \quad \tau_{i, t}=\tau_{i, t-1}+r M_{t}
$$

As usual, the precision hyper-parameter increases in time, provided that at least one consumer buys a positive quantity at date $t$ : the pace of increase is given by the objective precision $r$. On the other hand, the updated mean parameter can be viewed as a weighted average between its previous value and the last observed quality mean: the weights are, respectively, the previous value of subjective precision and the contribution of last observation to the increase of precision itself. It follows that $i$ 's opinion on the true mean changes more and more slowly as time elapses, due to the increase in precision. A high initial precision $\tau_{i, 0}$ means that $i$ is already very confident in her opinion at very early stages, and her rate of learning is slow.

It is however well-known ${ }^{14}$ that, if consumers keep accumulating observations (i.e. if $M_{t}>0$, all $t$ 's), then

$$
\operatorname{Prob}\left[\lim _{t \rightarrow \infty} \mu_{i, t}=\mu_{T}\right]=1
$$

This means that, if the innovation does not abort in finite time, all consumers learn its true quality in the limit, with precisions diverging to infinity. Under these circumstances, and given Assumption 1, they all buy asymptotically the same positive quantity for any given price announced by the firm. Such common quantity is $p^{\frac{1}{\delta-1}} K^{*}$, where $K^{*}$ is defined starting from expression (2.8), and setting $\left(\tau_{i, t}\right)^{-1}=0$ and $\mu_{i, t}=\mu_{T}$ for all $i$ 's; hence, the asymptotic market demand is $Q^{*}(p)=p^{\frac{1}{\delta-1}} M K^{*}$.

\section{$2.3 \quad$ Firm's choice and learning}

The firm launching the new product is the monopolist of this product, and it bears a constant marginal cost equal to $c$.

Not knowing the minds of potential buyers, the firm must conjecture a demand curve for the new product. To keep things as simple as possible, we

\footnotetext{
${ }^{13}$ See e.g. De Groot 1970, chapter 9.

${ }^{14}$ Due to some Law of large numbers.
} 
assume that it conjectures a linear ${ }^{15}$ market demand function; that is, the firm believes that, for any value of $a, b$ and $p$, the conditional distribution of the quantity demanded $Q$ is a normal variable with given precision, say 1 for simplicity, and mean $Q=a-b p$.

The firm, being uncertain about the values of $a$ and $b$, maintains the hypothesis that their distribution is a normal bivariate: the mean and the precision hyper-parameters of this distribution at date $t$ are as follows ${ }^{16}$ :

$$
\mathbf{m}_{t}=\left(\begin{array}{c}
\alpha_{t} \\
\beta_{t}
\end{array}\right) \quad \text { and } \quad \boldsymbol{\Gamma}_{t}=\left(\begin{array}{cc}
\gamma_{1, t} & \gamma_{12, t} \\
\gamma_{12, t} & \gamma_{2, t}
\end{array}\right)
$$

where $\alpha_{t}, \beta_{t}, \gamma_{1, t}$ and $\gamma_{2, t}$ are positive. Since the firm has surely no reason to conjecture any particular initial value for the correlation among the two mean hyper-parameters, we assume $\gamma_{12,0}=0$. For notational easiness we put $\gamma_{1,0} \equiv \gamma_{1}$ and $\gamma_{2,0} \equiv \gamma_{2}$. Call $f_{F, t}(a, b)$ firm's conjecture at date $t$.

This given, for any price $p_{t}$ to be announced at date $t$, the firm expects the following market demand:

$$
E_{F, t-1}\left(Q_{t} \mid p_{t}\right)=\alpha_{t-1}-\beta_{t-1} p_{t}
$$

where $E_{F, t-1}[\cdot]$ is taken w.r.t. $f_{F, t-1}(a, b)$. Notice, again, the timing: the firm announces the price before the resulting actual demand is observed, hence uses its $(t-1)$-conjecture, formed observing demand at time $(t-1)$.

The firm chooses the price so as to maximize expected profit, $E_{F, t-1}\left(Q_{t} \mid p_{t}\right)$. $\left(p_{t}-c\right)$. Therefore, the price announced at date $t$ is ${ }^{17}$

$$
p_{t}=\frac{\alpha_{t-1}}{2 \beta_{t-1}}+\frac{c}{2}=\frac{\alpha_{t-1}+c \beta_{t-1}}{2 \beta_{t-1}}
$$

and the resulting expected demand, $Q_{t}^{e}\left(p_{t}\right)$, is

$$
Q_{t}^{e}\left(p_{t}\right)=\frac{\alpha_{t-1}-c \beta_{t-1}}{2}
$$

\footnotetext{
${ }^{15} \mathrm{~A}$ non-linear formulation would clearly be more satisfactory: nothing substantial would however be added to the material that follows, and calculations would be uselessly cumbersome.

${ }^{16}$ This is a special case, deriving from our assumption that the conditional distribution of $Q$ given $a, b$ and $p$ has known precision equal to 1 ; if this precision were different from 1 , matrix $\Gamma_{t}$ would be multiplied by its value. Things could be generalized, but this would be immaterial for our results, since firm's expected profit does not depend on precisions.

${ }^{17}$ It must of course be the case that $\alpha_{t} / \beta_{t}>c$, otherwise the firm would not find it profitable to sell anything.
} 
We assume that the firm can meet all the demand that is coming at this price; if demand is lower than expected, the firm can either curtail production "just-in-time" or freely dispose of the excess supply.

Let us turn to learning. After actual demand ${ }^{18} Q_{t}^{D}$ is observed at $t \geq 1$, the firm updates the posterior distribution following Bayesian rules. Define the row vector ${ }^{19} \mathbf{x}_{t}^{\prime} \equiv\left[\begin{array}{cc}1 & -p_{t}\end{array}\right]$; given our assumptions, one has ${ }^{20}$ :

$$
\mathbf{m}_{t}=\left[\boldsymbol{\Gamma}_{t-1}+\mathbf{x}_{t} \mathbf{x}_{t}^{\prime}\right]^{-1}\left[\boldsymbol{\Gamma}_{t-1} \mathbf{m}_{t-1}+\mathbf{x}_{t} Q_{t}^{D}\right]
$$

and

$$
\boldsymbol{\Gamma}_{t}=\left[\boldsymbol{\Gamma}_{t-1}+\mathbf{x}_{t} \mathbf{x}_{t}^{\prime}\right]
$$

Expression (2.14) says that, being $\mathbf{x}_{t} \mathbf{x}_{t}^{\prime}$ a semi-positive definite matrix, precision always "increases" 21 in time. (2.13) can also be written as

$$
\begin{aligned}
\mathbf{m}_{t} & =\left[\boldsymbol{\Gamma}_{t-1}+\mathbf{x}_{t} \mathbf{x}_{t}^{\prime}\right]^{-1}\left[\left(\boldsymbol{\Gamma}_{t-1}+\mathbf{x}_{t} \mathbf{x}_{t}^{\prime}-\mathbf{x}_{t} \mathbf{x}_{t}^{\prime}\right) \mathbf{m}_{t-1}+\mathbf{x}_{t} Q_{t}^{D}\right]= \\
& =\left[\boldsymbol{\Gamma}_{t-1}+\mathbf{x}_{t} \mathbf{x}_{t}^{\prime}\right]^{-1}\left[\left(\boldsymbol{\Gamma}_{t-1}+\mathbf{x}_{t} \mathbf{x}_{t}^{\prime}\right) \mathbf{m}_{t-1}+\mathbf{x}_{t}\left(Q_{t}^{D}-\mathbf{x}_{t}^{\prime} \mathbf{m}_{t-1}\right)\right]= \\
& =\mathbf{m}_{t-1}+\left[\boldsymbol{\Gamma}_{t-1}+\mathbf{x}_{t} \mathbf{x}_{t}^{\prime}\right]^{-1}\left[\mathbf{x}_{t}\left(Q_{t}^{D}-\mathbf{x}_{t}^{\prime} \mathbf{m}_{t-1}\right)\right]
\end{aligned}
$$

meaning that the updated parameters $\mathbf{m}_{t}$ are equal to their previous values, plus a term involving the "prediction error" appearing in round brackets of $(2.15)^{22}$, "deflated" by the updated precision. Once more, then, we find that the firm's subjective opinion about the parameters to be estimated changes more and more slowly as time elapses, so that it converges asymptotically somewhere $^{23}$. We cannot, however, assume that the firm's opinion converges to any "true" value: as regards this point, see to section 4 below.

\subsection{The learning dynamical system}

The updating of posteriors on the part of agents gives rise to a dynamical system, whose evolution describes the diffusion path of the new product. We wish to write this system in a compact way, convenient for subsequent uses.

\footnotetext{
${ }^{18}$ See expression (2.9) above.

${ }^{19}$ In OLS terms, the two elements appearing in vector $\mathbf{x}_{t}$ are the "regressors" of the equation $Q_{t}^{D}=\alpha_{t}-\beta_{t} p_{t}$, which is estimated recursively.

${ }^{20}$ See De Groot [1970], chapter 11.

${ }^{21}$ In terms of the positive-definite-matrix ordering. As a consequence, since $\gamma_{1}$ and $\gamma_{2}$ are positive, $\Gamma_{t}$ is non-singular at all $t$.

${ }^{22}$ Notice that $\mathbf{x}_{t}^{\prime} \mathbf{m}_{t-1}$ in round brackets is expected demand $Q_{t}^{e}\left(p_{t}\right)$, as defined by (2.12).

${ }^{23}$ This happens if consumers' demand stays bounded, i.e. if the price does not go to zero, which is guaranteed by $c>0$.
} 
Define first $\boldsymbol{\mu}_{t}^{\prime}=\left[\begin{array}{lll}\mu_{1, t} & \ldots & \mu_{M, t}\end{array}\right]$ and $\boldsymbol{\tau}_{t}^{\prime}=\left[\begin{array}{lll}\tau_{1, t} & \ldots & \tau_{M, t}\end{array}\right]$, the vectors of all consumers' hyper-parameters at date $t$. Put in addition $\gamma_{t}^{\prime}=$ $\left[\begin{array}{llll}\gamma_{1, t} & \gamma_{12, t} & \gamma_{12, t} & \gamma_{2, t}\end{array}\right]$, the vectorization of matrix $\boldsymbol{\Gamma}_{t}$. Define finally the vector $\mathbf{y}_{t}^{\prime}=\left[\begin{array}{llll}\boldsymbol{\mu}_{t}^{\prime} & \boldsymbol{\tau}_{t}^{\prime} & \mathbf{m}_{t}^{\prime} & \gamma_{t}^{\prime}\end{array}\right]$. Thus, as shown in Appendix A, one can posit the following system of $(2 M+6)$ first order difference equations:

$$
\mathbf{y}_{t}=F\left(\mathbf{y}_{t-1}\right)
$$

which completely describes the diffusion dynamics.

\section{Failures and diffusion}

We address now the problem of diffusion. First, our interest is focused on factors that might hamper the diffusion of a product, albeit of good quality. Second, we explore the implications of our model as regards the logistic pattern of diffusion, considered as a stylized fact by many.

\subsection{Lock-in}

We ended Section 2.2 stating that if consumers keep accumulating observations their conjectures will converge to the true quality mean. However, it is clear that in any period the sample quality mean $\bar{\lambda}_{t}$ can be biased. We claim that a downward-biased signal can hamper the diffusion through the shrinking of the set of buyers, or can even drive aggregate demand to zero. Indeed, when heterogeneity is modeled as a difference in individual models, the pattern of learning depends both on initial conditions and on the particular set of information emerging on the path followed by the system. If some source of positive feedbacks exists, then a single piece of downwardbiased information might drive agents to rationally and definitively choose to be non-buyers, even if the true quality is high. In the present setup, positive feedbacks derive from the following two factors: first, the bayesian setting implies that precisions increase through time and reinforce existing opinions, whatever they are; second, if optimism (resp. pessimism) prevails, then a sustained number of buyers (resp. non-buyers) tends to feed everyone's opinion with a large (resp. poor) sample, this raising (resp. lowering) the probability that the true quality is discovered.

The lock-in literature stresses that the success/failure of an innovation is not decided in advance according to some fundamental parameter, e.g. the true quality mean: it may happen that a good product does not diffuse, or that a worse one does. We integrate this theory exploring the possibility 
that subjective factors may generate the result: a pessimistic initial constellation of conjectures can prevent diffusion, or, alternatively, the evolution of individual conjectures under downward-biased signals can harm it. This offers a new micro-foundation for the lock-in issue, grounded on rational choice under uncertainty and risk aversion: this foundation might complement those relying on some assumption of increasing returns, adopted e.g. in the famous Arthur's 1989 paper.

All technicalities supporting the points that follow are relegated to Appendix B. First, it is easily shown that "failure" is irreversible, that is $M_{t}=0$ implies $M_{t+1}=0$ (see Claim B.1). Second, we order consumers on the basis of an "optimism scale", induced by the index $\vartheta_{j, t}=\tau_{j, t}\left(\mu_{j, t}-\left(2 \tau_{j, t}\right)^{-1}-B\right)$, where $B=(2 r)^{-1}-\ln A$. Notice that, coherently with risk-aversion, "optimism" does not depend on the subjective mean parameter only, but also on the precision one. $\vartheta_{j, t-1} \leq 0$ implies a null demand on the part of $j$ at date $t$ (see expression B.1 in Appendix B); in addition lower values of $\vartheta_{j, t-1}$ imply a higher possibility of remaining, or becoming, a non-buyer at $t+1$ (see Remarks B.1). Third, conditions are derived under which the set of buyers shrinks or even vanishes, given the existing degree of optimism: these conditions boil down to the realization of a low enough sample quality mean (see Claims B.2, B.3 and B.4). Fourth, call $\vartheta_{t-1}^{+}$the optimism index of the most optimist buyer, which is necessarily positive: if $\vartheta_{t}^{+}$turns negative then the most optimist is pushed out of the market at date $t+1$, dragging all other buyers with her. This, as said above, implies irreversible failure $e^{24}$.

It is possible to evaluate the probability that failure takes place at date $t+1$, given that demand is positive at date $t$, and given $\vartheta_{t-1}^{+}$. In fact we have a main proposition, summarizing all previous results.

Proposition 1. Suppose that demand is positive at any time $\mathrm{t}$, that is $\vartheta_{t-1}^{+}>0$. Then the probability that demand becomes null at time $\mathrm{t}+1$ is:

$$
\operatorname{Pr}(\text { Failure })=\int_{-\infty}^{-\vartheta_{t-1}^{+}} \frac{1}{\sqrt{2 \pi M_{t} r}} \exp \left(-\frac{\left(v-M_{t} r\left(\mu_{T}-B\right)\right)^{2}}{2\left(M_{t} r\right)}\right) \mathrm{d} v>0
$$

Proof. See Appendix B

The probability of failure is always positive. It is decreasing in the true quality mean and, more interestingly, in $\vartheta_{t-1}^{+}$. Recalling that $\vartheta_{t-1}^{+}$incorpo-

\footnotetext{
${ }^{24}$ Ideally, one can imagine a succession of sample means that withdraw single buyers from the market sequentially in time; but the logic is the same: if aggregate demand reaches zero, because the most optimist buyer changes her status, then it remains zero forever.
} 
rates the buyer's uncertainty (measured inversely by $\tau_{j, t-1}$ ), this is a major result showing the role played by risk aversion in preventing the diffusion of a (high-quality) new product.

We can add something about the probability of failure. In fact Corollary B.1 of Appendix B shows that this probability is increasing in the number $M_{t}$ of adopters, provided that $M_{t}<\frac{\vartheta_{t-1}^{+}}{r\left(\mu_{T}-B\right)}$. This result is fairly intuitive: if the sample mean $\bar{\lambda}_{t}$ is low enough to turn optimist consumers into pessimist ones, this will be more effective if the biased sample mean is supported by a higher number of observations (see formulae 2.10). The condition $M_{t}<\frac{\vartheta_{t-1}^{+}}{r\left(\mu_{T}-B\right)}$ says in addition that such effect - that is, a greater number of buyers increases the probability of failure- is more permanent if the most optimist buyer is highly optimist, and/or if the objective precision $r$ is low. However, if the number of buyers gets higher than a certain threshold, an opposite and obvious effect takes place: namely, that the probability of very downward-biased sample means, and hence of failure, decreases.

\subsection{Product diffusion}

We proved that a new product, though of high quality, can fail to diffuse due to low consumers' initial expectations and/or to downward-biased samples. We assume now that the product does diffuse, and discuss the "transient dynamics", enquiring whether our model can predict the oft-observed $S$ shaped diffusion curve. Our attempt is to analyze the microfundamentals of such $S$-shaped curve in terms of heterogeneity and risk aversion. The latter implies that time, through the provision of information, increases individual precisions, and hence favors diffusion. At the same time, the speed of diffusion depends both on the learning rate, which again is determined by individual precisions, and on the constellation of individual conjectures, namely how far they are from the true mean.

Since the speed of consumer learning (the time-change of $\mu_{i, t}$ ) goes to zero as time goes to infinity, any increasing ${ }^{25}$ diffusion curve turns concave in the limit, for given price. Hence, in order to have a $S$-shaped curve it is enough to check that the rate of growth of market demand increases during some early stages. The growth rate of market demand, in turn, is a

\footnotetext{
${ }^{25}$ Obviously, one cannot exclude a decreasing diffusion path, if consumers' expectations are over-optimistic at the outset. Along lines similar to those of section 3.1, one could also prove that in this case there is a positive probability that market demand increases in the early stages; however, it must sooner or later converge down to $Q^{*}(p)$, as defined at the end of section 2.2. Here we concentrate on increasing paths.
} 
weighted average of individual demands' rates $^{26}$ : so it is enough to show that individual demands can be $S$-shaped. Obviously, individual demand could accelerate if the price went down: but, to grasp the bulk of the problem, we consider a fixed price ${ }^{27}$. To get rid of quality noise, we reason in expectedvalue terms, w.r.t. the distribution $f_{T}(\lambda)$ : consumers observe $\mu_{T}$ at each date. This is a standard procedure in logistic models.

Consider individual demand (2.5): given the price, its growth rate is proportional to that of $\left(A-e^{z_{t}}\right)$, having defined $z_{t} \equiv(2 r)^{-1}+\left(2 \tau_{t}\right)^{-1}-\mu_{t}$, and omitting the subscript $i$ for brevity. The rate of growth of $\left(A-e^{z_{t}}\right)$ is

$$
\frac{e^{z_{t}}-e^{z_{t+1}}}{A-e^{z_{t}}}
$$

Defining now $\Delta z \equiv z_{t+1}-z_{t}$, one can write this rate of growth as

$$
\frac{1-e^{\Delta z}}{\frac{A}{e^{z} t}-1}
$$

Since we are considering increasing diffusion paths, meaning $\mu_{t}<\mu_{T}, e^{z_{t}}$ clearly decreases in time, and $\Delta z<0^{28}$. Therefore, the denominator of (3.1) keeps increasing in time, and an increase of the growth rate, i.e. a $S$-shaped diffusion curve, can only come from a rapid increase of the numerator.

Consider thus the term $\Delta z$. Recalling the learning formulae (2.10), and setting $\bar{\lambda}_{t}=\mu_{T}$, after some manipulation one can write this term as

$$
\Delta z=\frac{r M_{t}}{\tau_{t}+r M_{t}}\left(\mu_{t}-\mu_{T}-\frac{1}{2 \tau_{t}}\right)
$$

It is apparent that $\Delta z$ increases rapidly if $\mu_{t}$ is significantly lower than the true mean $\mu_{T}$ : in this case, indeed, observing $\mu_{T}$ causes a wide change in $\mu_{t}$. One might think that a very low subjective precision $\tau_{t}$, also, fosters an increase of $\Delta z$ : in fact, if $\tau_{t}$ is low, $1 / 2 \tau_{t}$ decreases substantially at $t+1$. However, a very low precision makes $\mu_{t}$ converge suddenly to $\mu_{T}$, and thus prevents a sustained growth of $\mu_{t}$, necessary for a $S$-shaped diffusion curve.

The above arguments are given support by Figures $1 \mathrm{a}$ and $1 \mathrm{~b}$, where we set the following parameter values: $p=r=A=1, \delta=0.7, \mu_{T}=4$, and

\footnotetext{
${ }^{26}$ With a null weight given to cases satisfying (2.6).

${ }^{27}$ In our setting the firm has no incentive to follow a moving-price policy, lowering the price in the first stages to capture buyers: in fact individual demands remain null if (2.6) holds, even for very low prices. In addition, our setup ignores competition, an otherwise major source of decreasing price and hence diffusion.

${ }^{28}$ Recall learning formulae (2.10), setting $\bar{\lambda}_{t}=\mu_{T}$. Also, if the consumer buys a positive quantity, the denominator of (3.1) is positive: then $\Delta z<0$ implies that (3.1) is positive.
} 
$\tau_{0}=70$. We calculate total demand for 100 periods under the two cases $\mu_{0}=0.4$ and $\mu_{0}=1$ : as expected, the former case gives rise to a $S$-shaped curve, while the latter one does not. Figure 1c shows in addition that, if we reduce the initial precision $\tau_{0}$ from 70 to 10 while keeping $\mu_{0}=0.4$, the initial speed of learning increases, preventing the curve from being convex in the early stages.

Our model, then, predicts a $S$-shaped diffusion curve if consumers are mean-pessimist, but not very uncertain, at the outset.

\section{Conjectural equilibria and their stability}

\subsection{Characterization of conjectural equilibria}

We turn now to the problem of the long run behavior of our dynamical system (2.16), assuming that the innovation does not abort in early stages. To this end we introduce the following

Definition 1 (Conjectural equilibrium). A conjectural equilibrium (CE) is a $(M+1)$-vector $\mathbf{c}_{t} \equiv\left[\begin{array}{llll}q_{1, t} & \ldots & q_{M, t} & p_{t}\end{array}\right]$ of consumers' and firm's choices such that $\mathbf{c}_{t+1}=\mathbf{c}_{t}$, all $\mathrm{t}$, under the operation of dynamical system (2.16).

The reason why we use the term "conjectural" will become clear in a while. Let us explore first the conditions to be met in order that individual choices form a CE. On the one hand, consumers' demands $q_{i, t}$ remain constant in time if both hyper-parameters of their posteriors, together with price, are constant. Therefore, given consumer's learning mechanism (2.10), in order to have constant demands for given price one must consider the limiting case $f_{i, \infty}(\cdot) \equiv \lim _{t \rightarrow \infty} f_{i, t}(\cdot)$, for all $i$ 's: the precision diverges to infinity, and the believed mean has reached $\mu_{T}$. Therefore market demand is $Q^{*}(p)=p^{\frac{1}{\delta-1}} M K^{*}$, as defined at the end of section 2.2 .

On the other hand, the price remains constant in time if firm's mean hyper-parameters, $\mathbf{m}_{t}$, do not change ${ }^{29}$. From expression (2.15) we know that $\mathbf{m}_{t}$, in turn, does not change if and only if the firm's expectation of market demand is fulfilled, i.e. if $Q_{t}^{D}=Q_{t}^{e}=\mathbf{x}_{\mathbf{t}}{ }^{\prime} \mathbf{m}_{t-1}$. If this condition is satisfied the price and, hence, consumers' demand stay constant, so that firm's expectation keeps being fulfilled.

\footnotetext{
${ }^{29}$ In this case we need not consider the asymptotic case $\lim _{t \rightarrow \infty} f_{F, t}(\cdot)$, because firm's choice does not depend on the precision matrix $\boldsymbol{\Gamma}_{t}$.
} 
16 Quality Uncertainty, Risk Aversion, and New Product Diffusion

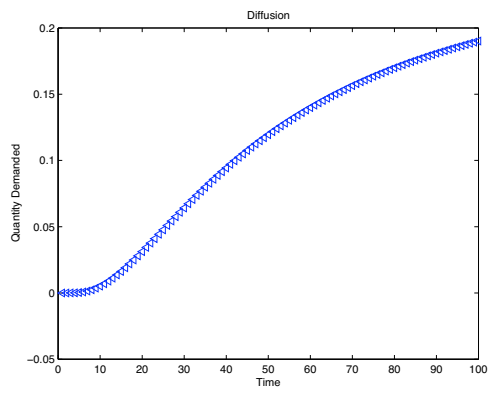

(a) $\mu_{0}=0.4$ and $\tau_{0}=70$

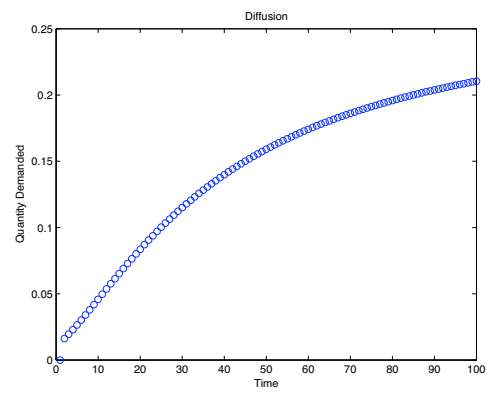

(b) $\mu_{0}=1$ and $\tau_{0}=70$

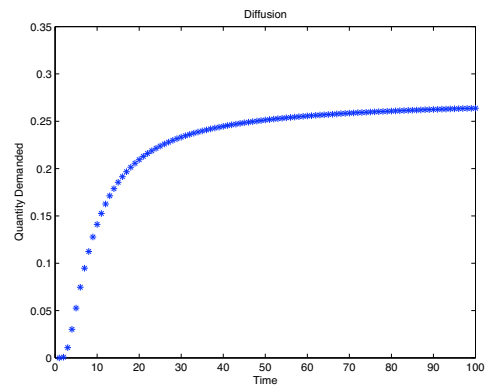

(c) $\mu_{0}=0.4$ and $\tau_{0}=10$

Figure 1: Some numerical examples of the of evolution of individual demand. 
Recalling definitions given in section 2 above $^{30}$, the $C E$ condition $Q_{t}^{D}=$ $Q_{t}^{e}$ can be expressed as follows:

$$
\left(\frac{\alpha^{*}+c \beta^{*}}{2 \beta^{*}}\right)^{\frac{1}{\delta-1}} M K^{*}=\frac{\alpha^{*}-c \beta^{*}}{2}
$$

together with $f_{i, t}(\cdot)=f_{i, \infty}(\cdot)$, all $i$ 's (asterisks denote equilibrium values).

It is now apparent why we speak of conjectural equilibrium. Indeed (4.1) defines a whole one-dimensional manifold (a curve) in the $(\alpha, \beta)$ space $^{31}$. That is, we have a continuum of CE's, depending on different configurations of the firm's (fulfilled) conjecture: there is not a unique position where our dynamical system can stay in the long run ${ }^{32}$. CE's differ among themselves as regards price and quantity, not the number of buyers nor perceived quality: the latter are equal to $M$ and $\mu_{T}$ in all equilibria.

As one may suspect, although in any CE the firm maximizes expected profits (given its conjecture), these profits may not be at their maximum level, as computed using the true demand curve: call Maximum True Profit Equilibrium, MTPE, this particular CE. An important feature of MTPE is tangency between the two demand curves. In fact, solving its problem, the firm prices where the Lerner Index $\frac{p-c}{p}$ is equal to the inverse of the elasticity of demand. Therefore MTPE requires $\frac{p-c}{p}=\frac{1}{\varepsilon_{T}}=\frac{1}{\varepsilon_{C}}$, where $\varepsilon_{T}$ and $\varepsilon_{C}$ are the true and the conjectured elasticities. It turns out that, given a conjecture $(\alpha, \beta)$, the inverse of the conjectured-demand elasticity is decreasing in price: $\frac{1}{\varepsilon_{C}}=\frac{\alpha}{\beta p}-1$; on the other side, the inverse of the true elasticity is constant: $\frac{1}{\varepsilon_{T}}=1-\delta$. In Figure 2 we see that there can exist only one point of intersection between the three curves (the Lerner Index, and the two inverse-elasticity curves, of which the conjectured one is parametrized by $\alpha$ and $\beta$ ).

Since the MTPE couple $\left(p^{*}, q^{*}\right)$ belongs to both the true and the conjectured demand (see equation 4.1), equality of elasticities implies tangency between the two curves. For given $c$ and $\delta$, the point where true profits are maximized is obviously unique: hence we can find just one $\alpha$ and one $\beta$ such that the linear conjectured demand is tangent to the hyperbole. Ergo, if a

\footnotetext{
${ }^{30}$ See also footnote 22 .

${ }^{31}$ Of course, not all mathematical solutions to (4.1) are economically sound.

${ }^{32}$ It is not easy to to find an explicit solution to the curve defined by (4.1). One can however characterize its linear approximation: defining $g(\alpha, \beta)=0$ by subtraction of the r.h.s. from the l.h.s. of (4.1), one finds that the partial derivatives of $g$ w.r.t. $\alpha$ and $\beta$ have opposite signs: see Appendix C, expression (C.5), for details. Then, by the implicit function theorem, one derives that the equilibrium relation between $\alpha$ and $\beta$ is increasing.
} 


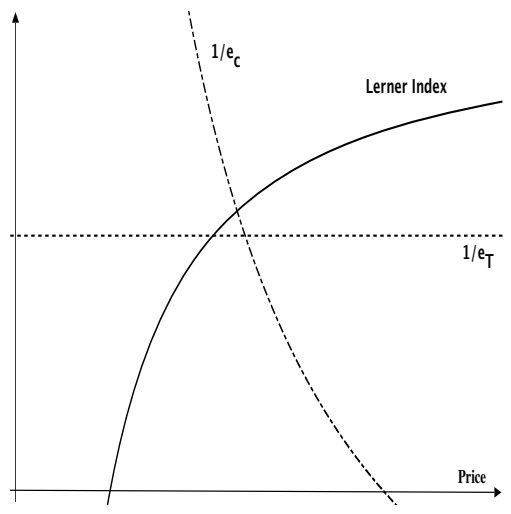

Figure 2: The elasticity rule: the Lerner Index, the inverse-elasticity of true demand, and one possible inverse-elasticity of equilibrium conjectured demand, all as functions of price.

MTPE exists, it is unique. In Appendix C we prove indeed Corollary C.1, stating that the MTPE exists.

Another interesting property of our setup is that we can make welfare analysis along the CE manifold defined by (4.1). As we discussed in Section 2.2 , we avoid here all the problems related with income effects, so we can use the sum of Consumer and Producer Surplus as a measure of welfare, following the standard partial equilibrium approach. The welfare effects of changes of the $\mathrm{CE}$ parameters $(\alpha, \beta)$ are completely captured by price. The proof of Corollary C.1 in Appendix C shows that along the CE manifold the conjectured demand becomes more and more elastic as $\alpha$ and $\beta$ increase. Hence the firm has a reduced ability to extract surplus, and it is forced to approach a competitive result. Said differently, since price decreases as $\alpha$ and $\beta$ increase (see the proof of Corollary C.3 in Appendix C), the surplus increases.

\subsection{Stability}

If, at any date, the system is in one of the states defined by (4.1), together with $f_{i, t}(\cdot)=f_{i, \infty}(\cdot)$, it will stay there forever. We want to address now the problem of stability: more precisely, we study local stability, i.e. ask whether an equilibrium can be reached starting form initial conditions "nearby" it. This requires evaluating the jacobian of system (2.16) at an equilibrium at 
a certain date $t$, namely

$$
J_{F, t}^{*}=\left[\frac{\partial y_{t+1}}{\partial y_{t}}\right]_{f_{i, \infty}, \alpha^{*}, \beta^{*}, t}
$$

and checking that none of its eigenvalues exceeds 1 in absolute value.

As it always happens in the presence of multiple equilibria, the asymptotic state of the system is sensitive to initial conditions: to each different initial condition there corresponds a different asymptotic state. In our present case, consumers' conjectures being fixed at their limiting positions $f_{i, \infty}(\cdot)$, the relevant initial condition is the firm's prior ${ }^{33}$.

Indeed it turns out that the local stability of a CE depends heavily on the firm's prior, besides depending on the elasticity of the true demand, as the following Proposition shows.

Proposition 2. Out of the $(2 M+6)$ eigenvalues of $J_{F, t}^{*}$, only one can exceed 1 in absolute value, and more precisely can be lower than -1, all the others being positive. This can happen if the following conditions are satisfied:

(i) the prior precision of the $\alpha$ parameter, $\gamma_{1}$, is low;

(ii) the prior precision of the $\beta$ parameter, $\gamma_{2}$, is lower than $\gamma_{1}$;

(iii) t is small, that is the firm is the early stages of learning;

(iv) the elasticity of the true demand, $\varepsilon_{T}$, is high relative to that conjectured by the firm, $\varepsilon_{C}$.

Proof. See Appendix C

We conclude that, even though the firm's prior were located near a CE, under the conditions of Proposition 2 the learning dynamics would push the variables away from that equilibrium if the firm is highly uncertain at the outset ${ }^{34}$ : uncertainty is embodied in low values of prior precisions. In particular, instability is reinforced by a high firm's uncertainty about by the steepness of demand. In addition, instability is greater for high elasticity values of the true demand, meaning that consumers react strongly (more strongly than the firm expects) to price changes. Coherently with time bringing about an increase in precisions, instability decreases in $t$ : if the

\footnotetext{
${ }^{33}$ If we considered any possible initial condition, i.e. $f_{i, t}(\cdot) \neq f_{i, \infty}(\cdot)$, the asymptotic state would of course depend also on the path followed by learning, that is on the sample realizations of quality. However, since in our setting consumers are bound to learn the true quality asymptotically, this would add nothing interesting about stability of equilibria.

${ }^{34}$ The path followed by the system while escaping from equilibrium displays oscillations, due to the negativity of the unstable eigenvalue.
} 
system were to pass nearby the same $\mathrm{CE}$ at a subsequent date, variables would no longer be pushed away from it.

From the proof of Proposition 2, it is apparent that we are speaking of Lyapunov, not asymptotic, stability. In fact, the very presence of a continuum of equilibria implies that moving away from a $\mathrm{CE}$ along the direction defined by (4.1) causes neither divergence from the new CE nor convergence to the previous one. This is explained in Claim C.2 of Appendix C.

Figure 3 gives an example of an unstable $\mathrm{CE}$. We locate the system nearby this CE at time $t=1$, and let the system run, taking $\mu_{i, t}=\mu_{T R U E}=$ $\bar{\lambda}_{t}$ to simulate consumers' certainty. The parameter values are: $t=1$, $\mu_{T R U E}=5, c=0, r=1, M=10, A=1, \delta=0.95, \gamma_{1}=1$, and $\gamma_{2}=0.0001$. The unstable eigenvalue is -23.9938 . It is apparent that, after initial divergence, the system becomes more stable in time, as predicted by our previous arguments.

Interestingly, the MTPE is stable: this is proved by Corollary C.2 in Appendix C.

In addition, Corollary C.3 in Appendix C proves that for low $t$ and high firm's uncertainty, when $\alpha$ and $\beta$ decrease (increase) along the CEmanifold equilibria become more unstable (stable). We already know that, along the same directions on the CE-manifold, conjectured demand becomes less (more) elastic and welfare decreases (increases): therefore stability and efficiency increase together.

One should not, however, conclude from this that less efficient equilibria are always fragile with respect to learning (as if "evolution selected the best"). In fact, the same inefficient equilibria are stable if the firm is more confident in its conjecture, and/or if enough time has elapsed already.

\section{Conclusions and extensions}

In this paper we have explored the market for a new product, formalizing the aspects of uncertainty that such a market involves: unknown quality on the demand side, unknown demand on the firm side. We have dealt explicitly with the heterogeneity issue: agents are endowed with different conjectures at the outset. Finally, on the demand side, we construed a setup to investigate the role played by risk aversion.

The main contributions of this paper relate to: (a) the "lock-in" theory, since we find conditions on subjective priors such that a biased signal can block the diffusion of a good product; (b) the study of the diffusion pattern, 


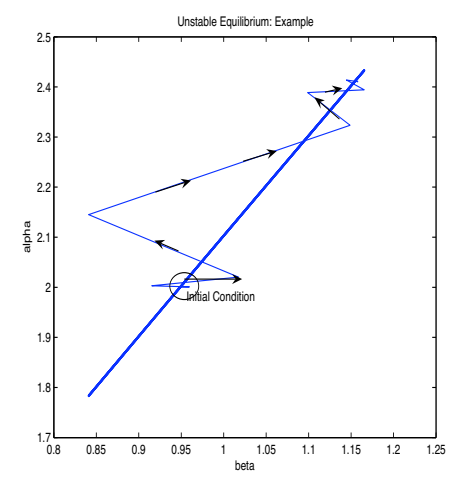

Figure 3: Shocking the system at an unstable equilibrium. The bold line is the equilibrium manifold, the thin line the trajectory of the firm shocked at an initial equilibrium.

since our model provides a micro-foundation for the $S$-shaped curve, but also shows the not-so-general character of this feature; (c) the equilibrium properties of the market, since coupling the two sides of the market generates a continuum of conjectural equilibria, with different welfare and stability properties.

Some generalizations are fairly natural and can be mentioned as directions for future work. First, the assumption that quality signals are publicly observable can be removed, introducing a cost for information acquisition. This may add a further source of lock-in, similar in spirit to the two-armedbandit literature ${ }^{35}$ : if the expected gain from new information is lower than its cost, agents can stop learning and get stuck into a bad choice.

Second, one might consider the possibility that the perceived quality depends on price, a point sometimes stressed by marketing researchers. One then expects that the true demand becomes more rigid and, as shown in Section 4 above, the set of stable conjectural equilibria is enlarged.

Third, the firm might be assumed to bear some initial investment cost, with the need to break even at some fixed date (due e.g. to capital market imperfections): if prior conjectures are pessimistic and if the diffusion curve is initially convex (see Section 3.2), a low demand in early stages may harm the success of the product, due to the firm's bankruptcy.

Fourth, one could assume that the information through which consumers update their posteriors is the quantity demanded, not only of the number

\footnotetext{
${ }^{35}$ See e.g. Rothschild (1974).
} 
of buyers. At a first sight, this would bring an important effect into the picture: indeed, even if the number of buyers is high, it might however happen that quantity demanded il low and this fact might prevent diffusion, a phenomenon which is not captured by our present model.

\section{References}

[1] Abrahamson E., Rosenkopf L. (1993), Institutional and competitive bandwagons: using mathematical modelling as a tool to explore innovation diffusion, Academy of Management Review, 18, 487-517

[2] Abrahamson E., Rosenkopf L. (1999), Modeling reputational and informational influences in threshold models of bandwagon innovation diffusion, Computational and Mathematical Organization Theory, 5, 361-384

[3] Agliardi E. (1998), Positive Feedback Economies, London, Macmillan

[4] Amable B. (1992), Effets d'apprentissage, compétitivité hors-prix et croissance cumulative, Economie Appliquée, vol. 45(3), 5-31

[5] Aoki M. and H. Yoshikawa (2002), Demand Saturation-Creation amd Economic Growth, Journal od Economic Behavior and Organization, 48, 127-154

[6] Arthur B. W. (1989), Competing Technologies, Increasing Returns, and Lock-in by Historical Events, Economic Journal, 99, March, 116-131

[7] Arthur B. W. (1994), Increasing Returns and Path Dependence in the Economy, Ann Arbor: University of Michigan Press

[8] Banerjee A., Fudenberg D. (2004) Word-of-mouth learning, Games and Economic Behavior, 46, 1-22

[9] Bass F. M. (1969) A new product growth model for consumer durables, Management Science, 15(5), 215-227

[10] Chatterjee R., Eliashberg J. (1990), The innovation diffusion process in a heterogeneous population: a micromodeling approach, Magament Science, 36, 1057-1079

[11] Cowan R., Jonard N., (2003), Network structure and the diffusion of knowledge, Journal of Economic Dynamics and Control, 28, 1557-1575

[12] Cowan R., Jonard N., (2004), The dynamics of collective invention, Journal of Economic Behavior and Organization, 52, 513-532

[13] Deffuant, G., S. Huet and F. Amblard (2005) An individual-based model of innovation diffusion mixing social value and individual benefit dynamics, American Journal of Sociology, Vol 110(4), 1041-1069

[14] De Groot M. H. (1970), Optimal Statistical Decision, New York, McGraw Hill 
[15] Dodson J.A. Jr., Muller E. (1978) Models of New Product Diffusion Through Advertising and Word-of-Mouth, Management Science, Vol. 24, 1568-1578

[16] Feder G., O'Mara G.T. (1982), On information and innovation diffusion: a Bayesian approach, American Journal of Agricultural Economics, 64, 145-147

[17] Gerosky P.A. (2000), Models of technology diffusion, Research Policy, 29, 603-25

[18] Hall, B.H. (2005) Innnovation and Diffusion, in J. Fagerberg, D. C. Mowery and R. R. Nelson, The Oxford Handbook of Innovation, pag 459-485

[19] Hivner W., Hopkins W.E., Hopkins S.A. (2003), Facilitating, accelerating and sustaining the innovation diffusion process: an epidemic modeling approach, European Journal of Innovation Management, 6, 80-89

[20] Ho T.-H., Savin S., Terwiesch C. Managing Demand and Sales Dynamics in New Product Diffusion Under Supply Constraint, Management Science, 48(2), 187-206

[21] Jackson M. O., Yariv L. (2007) Diffusion of Behavior and Equilibrium Properties in Network Games, American Economic Review, 97(2), 92-98

[22] Jensen R. (1982), Adoption and diffusion of an innovation of uncertain profitability, Journal of Economic Theory, 27, 182-193

[23] Lopez Pintado D., Watts D. J. (2006) Social Influence, Binary Decisions and Collective Dynamics, working paper

[24] Mahajan V., Peterson R.A. (1978), Innovation diffusion in dynamic a potential adopter population, Management Science, 15, 1589-97

[25] Mahajan V., Peterson R.A. (1985) Models of Innovation Diffusion, Springer

[26] Mukoyama T. (2006) Rosenberg's "Learning by Using" and technology diffusion, Journal of Economic Behavior and Organization, 61, 123-144

[27] Rampa G. (2002) Verdoorn's Law: Some Notes on Output Measurement and the Role of Demand, in J. McCombie, M. Pugno, B. Soro (eds), Productivity, Growth and Economic Performance: Essays on Verdoorn's Law, London, Palgrave, 2002, $219-238$

[28] M. Rothschild M. (1974) A two-armed bandit theory of market pricing, Journal of Economic Theory, 9, 185-202

[29] Tsur Y., Sternberg M., Ochman E. (1990), Dynamic modelling of innovation process adoption with risk aversion and learning, Oxford Economic Papers, 42, 336-355

[30] Verbrugge R. (2000), Risk Aversion, Learning Spillovers, and Path-dependent Economic Growth, Economics Letters, 68, 197-202

[31] Young P. (2005) The Spread of Innovation through Social Learning, CSED working paper $12 / 2005$ 
[32] Young P. (2007) Innovation Diffusion in Heterogeneous population, Economic Series Working Papers, 303, University of Oxford, Department of Economics

\section{Appendix}

\section{A Derivation of system (2.16)}

As regards consumers, expression (2.10) summarizes completely their learning. In order to represent firm's learning conveniently, start from expression (2.15) and notice that from the definition of vector $\mathbf{x}_{t}$ one derives

$$
\mathbf{x}_{t} \mathbf{x}_{t}^{\prime}=\left[\begin{array}{cc}
1 & -p_{t} \\
-p_{t} & p_{t}^{2}
\end{array}\right]
$$

We know from (2.11) that the price announced by the firm at date $t$ depends on the parameters $\mathbf{m}_{t-1}$, whence $\mathbf{x}_{t}=\mathbf{x}_{t}\left(\mathbf{m}_{t-1}\right)$ : one can thus write the term within the first square brackets of (2.15), i.e. the matrix to be inverted, as

$$
\left[\boldsymbol{\Gamma}_{t-1}+\mathbf{x}_{t} \mathbf{x}_{t}^{\prime}\right]=\left[\boldsymbol{\Gamma}_{t-1}+\left[\begin{array}{cc}
1 & -p_{t} \\
-p_{t} & p_{t}^{2}
\end{array}\right]\right] \equiv \mathbf{A}\left(\boldsymbol{\Gamma}_{t-1}, \mathbf{m}_{t-1}\right)
$$

On the other side, the term appearing within the second square brackets of (2.15) is the product of the column vector $\mathbf{x}_{t}$ and the scalar $\left(Q_{t}^{D}-\mathbf{x}_{t}^{\prime} \mathbf{m}_{t-1}\right)$; then one checks easily that it can be written as

$$
\left[\mathbf{x}_{t}\left(Q_{t}^{D}-\mathbf{x}_{t}^{\prime} \mathbf{m}_{t-1}\right)\right]=\left[\begin{array}{cc}
1 & 0 \\
0 & p_{t}
\end{array}\right]\left[\begin{array}{c}
\left(Q_{t}^{D}-\mathbf{x}_{t}^{\prime} \mathbf{m}_{t-1}\right) \\
-\left(Q_{t}^{D}-\mathbf{x}_{t}^{\prime} \mathbf{m}_{t-1}\right)
\end{array}\right]
$$

From (2.8) and (2.9) we know that market demand $Q_{t}^{D}$ depends on $p_{t}\left(\mathbf{m}_{t-1}\right)$ and on the posteriors of all consumers at date $(t-1)$. Therefore, defining

$$
\boldsymbol{\mu}_{t-1}^{\prime}=\left[\begin{array}{lll}
\mu_{1, t-1} & \ldots & \mu_{M, t-1}
\end{array}\right] \text { and } \boldsymbol{\tau}_{t-1}^{\prime}=\left[\begin{array}{lll}
\tau_{1, t-1} & \ldots & \tau_{M, t-1}
\end{array}\right]
$$

one can write $Q_{t}^{D}=Q_{t}^{D}\left(\mathbf{m}_{t-1}, \boldsymbol{\mu}_{t-1}, \boldsymbol{\tau}_{t-1}\right)$.

Collecting all the above material, (2.15) becomes

$$
\mathbf{m}_{t}=\mathbf{m}_{t-1}+\mathbf{C}\left(\boldsymbol{\Gamma}_{t-1}, \mathbf{m}_{t-1}\right) \cdot \mathbf{g}\left(\mathbf{m}_{t-1}, \boldsymbol{\mu}_{t-1}, \boldsymbol{\tau}_{t-1}\right)
$$

where

$$
\mathbf{C}\left(\boldsymbol{\Gamma}_{t-1}, \mathbf{m}_{t-1}\right) \equiv\left[\mathbf{A}\left(\boldsymbol{\Gamma}_{t-1}, \mathbf{m}_{t-1}\right)\right]^{-1} \mathbf{B}\left(\mathbf{m}_{t-1}\right), \quad \mathbf{B}\left(\mathbf{m}_{t-1}\right) \equiv\left[\begin{array}{cc}
1 & 0 \\
0 & p_{t}\left(\mathbf{m}_{t-1}\right)
\end{array}\right]
$$

and

$$
\mathbf{g}\left(\mathbf{m}_{t-1}, \boldsymbol{\mu}_{t-1}, \boldsymbol{\tau}_{t-1}\right) \equiv\left[\begin{array}{c}
\left(Q_{t}^{D}(\cdot)-\mathbf{x}_{t}^{\prime} \mathbf{m}_{t-1}\right) \\
-\left(Q_{t}^{D}(\cdot)-\mathbf{x}_{t}^{\prime} \mathbf{m}_{t-1}\right)
\end{array}\right]
$$


$\mathbf{C}(\cdot)$ is a 2 by 2 matrix, and $\mathbf{g}(\cdot)$ is a column 2 -vector. Now define

$$
\boldsymbol{\gamma}_{t}=\operatorname{vec}\left(\boldsymbol{\Gamma}_{t}\right)=\left[\begin{array}{llll}
\gamma_{1, t} & \gamma_{12, t} & \gamma_{12, t} & \gamma_{2, t}
\end{array}\right]
$$

Therefore, the updating of firm's precisions can be written as follows:

$$
\gamma_{t}=\gamma_{t-1}+\mathbf{v}\left(\mathbf{m}_{t-1}\right)
$$

where

$$
\mathbf{v}\left(\mathbf{m}_{t-1}\right) \equiv \operatorname{vec}\left(\mathbf{x}_{t} \mathbf{x}_{t}^{\prime}\right)=\left[\begin{array}{llll}
1 & -p_{t}\left(\mathbf{m}_{t-1}\right) & -p_{t}\left(\mathbf{m}_{t-1}\right) & p_{t}^{2}\left(\mathbf{m}_{t-1}\right)
\end{array}\right]^{\prime}
$$

Collecting (2.10), (A.2) and (A.3), and replacing $\boldsymbol{\Gamma}$ with $\gamma$ wherever it appears, we are eventually in a position of writing the learning dynamical system as follows:

$$
\begin{array}{rlr}
\mu_{i, t} & =\frac{\tau_{i, t-1} \mu_{i, t-1}+r M_{t} \bar{\lambda}_{t}}{\tau_{i, t-1}+r M_{t}} & i=1, \ldots, M \\
\tau_{i, t} & =\tau_{i, t-1}+r M_{t} & i=1, \ldots, M \\
\mathbf{m}_{t} & =\mathbf{m}_{t-1}+\mathbf{C}\left(\boldsymbol{\gamma}_{t-1}, \mathbf{m}_{t-1}\right) \cdot \mathbf{g}\left(\mathbf{m}_{t-1}, \boldsymbol{\mu}_{t-1}, \boldsymbol{\tau}_{t-1}\right) & \\
\boldsymbol{\gamma}_{t} & =\gamma_{t-1}+\mathbf{v}\left(\mathbf{m}_{t-1}\right) &
\end{array}
$$

Expressions (A.4)-(A.7) describe a system of $(2 M+6)$ first-order difference equations. Defining the $(2 M+6)$-vector $\mathbf{y}_{t}^{\prime}=\left[\begin{array}{llll}\boldsymbol{\mu}_{t}^{\prime} & \boldsymbol{\tau}_{t}^{\prime} & \mathbf{m}_{t}^{\prime} & \gamma_{t}^{\prime}\end{array}\right]$, we end up with the following notation:

i.e. our expression (2.16).

$$
\mathbf{y}_{t}=F\left(\mathbf{y}_{t-1}\right)
$$

\section{B Proof of Proposition 1}

Consider condition (2.6) for zero demand. Taking logarithms and defining

$$
B \equiv(2 r)^{-1}-\ln A \quad \text { and } \quad x_{j, t} \equiv \mu_{j, t}-\left(2 \tau_{j, t}\right)^{-1}
$$

we rewrite the condition for a null demand of consumer $j$ at date $t$ as

$$
j \notin C_{t} \Leftrightarrow x_{j, t-1} \leq B
$$

The set $C_{t}$, whose cardinality is $M_{t}$, was defined in section 2.1. Recall that demand at date $t$ depends on the posterior formed at date $t-1$.

Consider now consumer's learning rule (2.10). Given our definition of $x_{j, t}$, with some manipulation that rule can be transformed into

$$
x_{j, t}=\frac{\tau_{j, t-1}}{\tau_{j, t-1}+r M_{t}} x_{j, t-1}+\frac{r M_{t}}{\tau_{j, t-1}+r M_{t}} \bar{\lambda}_{t}
$$

where $\bar{\lambda}_{t}$, defined in section 2.1 , is the quality sample mean. We assume that, if no quality is observed (i.e. $M_{t}=0$ ), $\bar{\lambda}_{t}$ is conventionally set equal to the previous value of the hyper-parameter.

The proof of Proposition 1 goes through some steps. First of all we have a simple result, stating that failure is irreversible. 
Claim B.1. $\left(M_{t}=0\right) \Rightarrow\left(M_{t+s}=0\right)$ for all positive integers $\mathrm{s}$.

Proof. Trivial: $M_{t}=0$ means that (B.1) holds for all $j$ 's. But $\left(M_{t}=0\right)$ and (B.2), taken together, imply $x_{j, t}=x_{j, t-1}$ : hence (B.1) holds for all $j$ 's at $(t+1)$ as well, that is $M_{t+1}=0$. The Claim follows by induction.

We enquire next the condition under which consumer $j$ does not buy anything at date $t+1$, i.e. $j \notin C_{t+1}$. We offer the following

Claim B.2. One has $j \notin C_{t+1}$ if and only if

$$
\bar{\lambda}_{t} \leq B-\frac{1}{r M_{t}} \tau_{j, t-1}\left(x_{j, t-1}-B\right)
$$

Proof. $j \notin C_{t+1}$ means that (B.1) holds at date $(t+1)$, namely $x_{j, t} \leq B$. Using (B.2), this requirement leads to

$$
\tau_{j, t-1} x_{j, t-1}+r M_{t} \bar{\lambda}_{t} \leq B\left(\tau_{j, t-1}+r M_{t}\right)
$$

Rearranging terms, one obtains (B.3).

Some remarks are in order.

\section{Remarks B.1}

(a) Condition (B.3) is valid for both adopters and non-adopters at date $t$, that is independently of whether $j$ belongs to $C_{t}$ or not. The difference between the two lies in the sign of the term $\left(x_{j, t-1}-B\right)$ appearing in (B.3): for adopters it is positive, while it is negative for non-adopters, meaning that (B.3) is obviously harder to be satisfied for (previous) adopters.

(b) The quantity $\tau_{j, t-1}\left(x_{j, t-1}-B\right)$ appearing in (B.3) can be seen as a sort of "optimism indicator" on the part of consumer $j$, to be interpreted with sign as a propensity to adopt or not in the future. In fact, a negative value of $\left(x_{j, t-1}-B\right)$ -meaning non-adoption- together with a high value of $\tau_{j, t-1}$-meaning as we know a slow learning rate- makes (B.3) fairly easy to be satisfied: $j$ remains most probably a non-adopter. The opposite is true for positive values of $\left(x_{j, t-1}-B\right)$, together with high values of $\tau_{j, t-1}: j$ remains most probably an adopter. Finally, smaller values of $\tau_{j, t-1}$, for given negative (resp. positive) values of $\left(x_{j, t-1}-B\right)$, lower (resp. raise) the upper bound for $\bar{\lambda}_{t}$ in the non-adoption (resp. adoption) case, that is, they lower (resp. raise) the possibility of remaining (resp. becoming) a non-adopter.

(c) If the number of adopters $M_{t}$ decreases, the upper bound for $\bar{\lambda}_{t}$ in (B.3) decreases for adopters, and increases for non-adopters; that is, it is harder for both to change status.

Taking advantage of Remark (b) above, define $\vartheta_{j, t} \equiv \tau_{j, t}\left(x_{j, t}-B\right)$, and recall that $\vartheta_{j, t}$ is positive only for adopters. This variable can be used to define a "optimism" ordering $\prec_{\vartheta, t}$ of consumers, such that

$$
i \prec_{\vartheta, t} j \Leftrightarrow \vartheta_{i, t}<\vartheta_{j, t}
$$


Define now $j^{*}(t)$ such that $\left(j^{*}(t) \in C_{t}\right) \wedge\left(\forall j, \vartheta_{j, t} \geq 0: j^{*}(t) \prec_{\vartheta, t} j\right): j^{*}(t)$ is the least optimist consumer still buying a positive quantity at $t+1$. Define also $j^{+}(t)$ as the most optimist consumer, i.e. such that $\nexists j: j^{+}(t) \prec_{\vartheta, t} j$.

All this given, we can easily derive the following Claims B.3 and B.4.

Claim B.3. Suppose $C_{t} \neq \emptyset$ : then $C_{t+1} \subset C_{t}$ (strictly) if and only if (B.3) holds for $j=j^{*}(t-1)$, or for some $j$ such that $j^{*}(t-1) \prec_{\vartheta, t-1} j$.

Claim B.4. Suppose $C_{t} \neq \emptyset$ : then $C_{t+1}=\emptyset$ if and only if (B.3) holds for $j=$ $j^{+}(t-1)$.

Proof. Claim B.3 is a straightforward implication of condition (B.3), considering the ordering (B.4) and the definition of $j^{*}(t-1)$. Claim B.4 is a corollary of Claim B.3 and of the definition of $j^{+}(t-1)$.

Claim B.3 asserts that the set of buyers can contract in time; Claim B.4 says that this set can become empty, implying $M_{t+1}=0$ and hence irreversible failure. We want now to evaluate the probability that this last event occurs. To this end, define $\vartheta_{t-1}^{+} \equiv \vartheta_{j^{+}(t-1), t-1}$, the optimism indicator of the most optimist consumer.

Proposition 1. Suppose that demand is positive at any time $\mathrm{t}$, that is $\vartheta_{t-1}^{+}>0$. Then the probability that demand becomes null at time $\mathrm{t}+1$ is:

$$
\operatorname{Pr}(\text { Failure })=\int_{-\infty}^{-\vartheta_{t-1}^{+}} \frac{1}{\sqrt{2 \pi M_{t} r}} \exp \left(-\frac{\left(v-M_{t} r\left(\mu_{T}-B\right)\right)^{2}}{2\left(M_{t} r\right)}\right) \mathrm{d} v>0
$$

Proof. Given Claim B.4 the failure event corresponds to:

$$
\bar{\lambda}_{t} \leq B-\frac{1}{M_{t} r} \vartheta_{t-1}^{+}
$$

which can be rearranged as:

$$
M_{t} r\left(\bar{\lambda}_{t}-B\right) \leq-\vartheta_{t-1}^{+}
$$

Individual signals $\lambda_{j, t}$ are distributed as i.i.d. $N\left(\mu_{T}, r^{-1}\right)$, so $-M_{t}$ being the sample size- one has $\bar{\lambda}_{t} \sim N\left(\mu_{T},\left(M_{t} r\right)^{-1}\right)$. Then $M_{t} r\left(\bar{\lambda}_{t}-B\right) \sim N\left(M_{t} r\left(\mu_{T}-B\right), M_{t} r\right)$. The probability of condition (B.6) is thus the integral (B.5), and positivity follows from the normality assumption.

Corollary B.1. For high values of $\vartheta_{t-1}^{+}$and low values of $r$, the probability of failure is increasing in the number of adopters if such number is lower than $\frac{\vartheta_{t-1}^{+}}{r\left(\mu_{T}-B\right)}$.

Proof. Given the argument after (B.6) in the Proof of Proposition 1, one has:

$$
\operatorname{Pr}(\text { Failure })=\Phi\left(\frac{-\vartheta_{t-1}^{+}-M_{t} r\left(\mu_{T}-B\right)}{\sqrt{M_{t} r}}\right)
$$


where $\Phi(\cdot)$ is the Cumulative Density Function of $z \sim N(0,1)$. Hence, the probability of failure is increasing in $M_{t}$ if the argument of $\Phi(\cdot)$ has the same property.

Write the argument of $\Phi(\cdot)$ as $\frac{-\vartheta_{t-1}^{+}}{\sqrt{M_{t} r}}-\sqrt{M_{t} r}\left(\mu_{T}-B\right)$ : recall that $\vartheta_{t-1}^{+}>0$ if some optimist consumer exists at date $t-1$, and that Assumption 1 implies $\left(\mu_{T}-B\right)>0$. Define $w \equiv \sqrt{M_{t} r}>0$, and $u=\frac{-\vartheta_{t-1}^{+}}{w}-w\left(\mu_{T}-B\right): u$ is the argument of $\Phi(\cdot)$. The derivative of $u$ w.r.t. $w$ is equal to $\frac{\vartheta_{t-1}^{+}}{w^{2}}-\left(\mu_{T}-B\right)$ : this derivative is positive if $w^{2}<\frac{\vartheta_{t-1}^{+}}{\left(\mu_{T}-B\right)}$. Given the definition of $w$, it follows finally that $u$, and hence the probability of failure, is increasing in $M_{t}$ if $M_{t}<\frac{\vartheta_{t-1}^{+}}{r\left(\mu_{T}-B\right)}$.

\section{Proof of Proposition 2 and of Corollaries C.1-3}

Recalling (A.4)-(A.7) of Appendix A, the jacobian of system (2.16) is

$$
J_{F}=\left[\begin{array}{cc|cc}
{\left[\frac{\partial \mu_{i, t}}{\partial \mu_{j, t-1}}\right]} & {\left[\frac{\partial \mu_{i, t}}{\partial \tau_{j, t-1}}\right]} & \mathbf{0}_{M, 2} & \mathbf{0}_{M, 4} \\
\mathbf{0}_{M, M} & \mathbf{I}_{M} & \mathbf{0}_{M, 2} & \mathbf{0}_{M, 4} \\
\hline\left[\frac{\partial m_{k, t}}{\partial \mu_{j, t-1}}\right] & {\left[\frac{\partial m_{k, t}}{\partial \tau_{j, t-1}}\right]} & {\left[\frac{\partial m_{k, t}}{\partial m_{l, t-1}}\right]} & {\left[\frac{\partial m_{k, t}}{\partial \gamma_{s, t-1}}\right]} \\
\mathbf{0}_{4, M} & \mathbf{0}_{4, M} & {\left[\frac{\partial \gamma_{s, t}}{\partial m_{k, t-1}}\right]} & \mathbf{I}_{4}
\end{array}\right] \equiv\left[\begin{array}{c|c}
J_{1,1} & J_{1,2} \\
\hline J_{2,1} & J_{2,2}
\end{array}\right]
$$

where: $i, j=1, \ldots, M ; k, l=1,2 ; s=1, \ldots, 4 ; \mathbf{I}_{m}$ is the identity matrix of size $m$; and $\mathbf{0}_{m, n}$ is a $m$ by $n$ null matrix.

Now we evaluate this jacobian matrix in a conjectural equilibrium (as defined in section 4$), J_{F, t}^{*}$, and investigate its eigenvalues. The proof of Proposition 2 requires some steps, which we formalize through Claims (C.1)-(C.3).

Claim C.1. The first $2 M+4$ eigenvalues of $J_{F, t}^{*}$ are equal to 1 ; the remaining two ones are those of $\left[\frac{\partial m_{k, t}}{\partial m_{l, t-1}}\right]$.

Proof. $J_{F}$ is clearly decomposable, so its eigenvalues are those of $J_{1,1}(2 M$ in number) and those of $J_{2,2}$ (six in number). $J_{1,1}$ is decomposable as well, and its eigenvalues are the eigenvalues of $\mathbf{I}_{M}$, i.e. $M$ ones, plus those of $\left[\frac{\partial \mu_{i, t}}{\partial \mu_{j, t-1}}\right], i, j=1, \ldots, M$. Consider now expressions (A.4) and (A.5), and recall that in a conjectural equilibrium one has $M_{t}=M$ and $f_{i, t}(\cdot)=f_{i, \infty}(\cdot)$, as explained in section 4 . We have thus $\frac{\partial \mu_{i, t}}{\partial \mu_{i, t-1}}=\frac{\tau_{i, t-1}}{\tau_{i, t}}$, and $\frac{\partial \mu_{i, t}}{\partial \mu_{j, t-1}}=0$ for $i \neq j$, so that $\left[\frac{\partial \mu_{i, t}}{\partial \mu_{j, t-1}}\right]$ is a diagonal matrix. Letting $t \rightarrow \infty$, all precisions $\tau_{i, t}$ diverge to infinity, and hence $\frac{\tau_{i, t-1}}{\tau_{i, t}}$ converges to 1 from below, $\forall i$. All eigenvalues of $\left[\frac{\partial \mu_{i, t}}{\partial \mu_{j, t-1}}\right]$ are thus equal to one.

Let us pass to $J_{2,2}$, and consider its "north-east" block $\left[\frac{\partial m_{k, t}}{\partial \gamma_{s, t-1}}\right]$. Collecting the 
definition in (A.1), those after (A.2), and equation (A.6), one can write that block in matrix form as:

$$
\frac{\partial \mathbf{C}(\cdot)}{\partial \boldsymbol{\gamma}_{t-1}} \mathbf{g}(\cdot)+\mathbf{C}(\cdot) \frac{\partial \mathbf{g}(\cdot)}{\partial \boldsymbol{\gamma}_{t-1}}
$$

The first term of last expression is null owing to the definition of equilibrium: in fact, from the definition of $\mathbf{g}(\cdot)$ after (A.2), and from the equilibrium condition discussed with reference to expression $(4.1)$, one gets $\mathbf{g}(\cdot)=0$ at equilibrium. The second term of the last expression is null because $\mathbf{g}(\cdot)$ does not depend on $\gamma_{t-1}$. Hence, the "north-east" block of $J_{2,2}$ is null, and $J_{2,2}$ is decomposable into $\left[\frac{\partial m_{k, t}}{\partial m_{l, t-1}}\right]$ and $\mathbf{I}_{4}$.

Therefore, four of its eigenvalues are equal to 1, while the last two are those of $\left[\frac{\partial m_{k, t}}{\partial m_{l, t-1}}\right]$. The Claim is proved.

The meaning of the $M+4$ unitary eigenvalues, relating to $\mathbf{I}_{M}$ and $\mathbf{I}_{4}$, is that consumers' and firm's precisions do not converge (indeed they diverge to infinity), without however impeding the convergence of the mean parameters $\mu_{i, t}$ and $\mathbf{m}_{t-1}$. The other $M$ eigenvalues, converging to 1 from below, mean that parameters $\mu_{i, t}$ get closer and closer to their limiting value $\mu_{T}$ at a decreasing speed.

It follows that stability depends entirely on the eigenvalues of $\left[\frac{\partial m_{k, t}}{\partial m_{l, t-1}}\right]$. We have the following result.

Claim C.2. ( $i)$ One of the two eigenvalues of $\left[\frac{\partial m_{k, t}}{\partial m_{l, t-1}}\right]$ is equal to 1 ;

(ii) the eigenvector associated with the above unitary eigenvalue is the linear approximation to the equilibrium manifold, defined by equation $(4.1)$, at the $(\alpha, \beta)$ point defining a conjectural equilibrium;

(iii) the other eigenvalue of $\left[\frac{\partial m_{k, t}}{\partial m_{l, t-1}}\right]$ is equal to 1 plus the trace of matrix $\mathbf{C}(\cdot) \frac{\partial \mathbf{g}(\cdot)}{\partial \boldsymbol{m}_{t-1}}$.

Proof. (i) We write $\left[\frac{\partial m_{k, t}}{\partial m_{l, t-1}}\right]$ in matrix form, which is easily checked to be

$$
\left[\frac{\partial m_{k, t}}{\partial m_{l, t-1}}\right]=\mathbf{I}_{2}+\frac{\partial \mathbf{C}(\cdot)}{\partial \boldsymbol{m}_{t-1}} \mathbf{g}(\cdot)+\mathbf{C}(\cdot) \frac{\partial \mathbf{g}(\cdot)}{\partial \boldsymbol{m}_{t-1}}=\mathbf{I}_{2}+\mathbf{C}(\cdot) \frac{\partial \mathbf{g}(\cdot)}{\partial \boldsymbol{m}_{t-1}}
$$

The last passage derives again from being $\mathbf{g}(\cdot)=0$ in equilibrium. Hence the two eigenvalues of $\left[\frac{\partial m_{k, t}}{\partial m_{l, t-1}}\right]$ are equal to 1 plus the eigenvalues of $\mathbf{C}(\cdot) \frac{\partial \mathbf{g}(\cdot)}{\partial \boldsymbol{m}_{t-1}}$. Consider now matrix $\frac{\partial \mathbf{g}(\cdot)}{\partial \boldsymbol{m}_{t-1}}$. As $\mathbf{g}(\cdot)$ if clearly of the form $\left[\begin{array}{ll}v & -v\end{array}\right]^{\prime}$, matrix $\frac{\partial \mathbf{g}(\cdot)}{\partial \boldsymbol{m}_{t-1}}$ is singular. Therefore $\mathbf{C}(\cdot) \frac{\partial \mathbf{g}(\cdot)}{\partial \boldsymbol{m}_{t-1}}$ is singular as well, so that one of its eigenvalues is zero, and thus one one the eigenvalues of $\left[\frac{\partial m_{k, t}}{\partial m_{l, t-1}}\right]$ is equal to 1 .

(ii) Since all vectors are eigenvectors of $\mathbf{I}_{2}$, the eigenvector of $\left[\frac{\partial m_{k, t}}{\partial m_{l, t-1}}\right]$ associated 
with this unitary eigenvalue is the same as the eigenvector of $\mathbf{C}(\cdot) \frac{\partial \mathbf{g}(\cdot)}{\partial \boldsymbol{m}_{t-1}}$ associated with its null eigenvalue. Take the equilibrium condition (4.1), and write it as

$$
g(\alpha, \beta) \equiv\left(\frac{\alpha+c \beta}{2 \beta}\right)^{\frac{1}{\delta-1}} M K-\frac{\alpha-c \beta}{2}=0
$$

It is clear that the last expression is the first element of vector $\mathbf{g}(\cdot)$ (and, changing the sign, it is its second element). Therefore the eigenvector of of $\mathbf{C}(\cdot) \frac{\partial \mathbf{g}(\cdot)}{\partial \boldsymbol{m}_{t-1}}$ associated with its null eigenvalue is nothing else than the vector orthogonal to the gradient of $g(\alpha, \beta)$. By the implicit function theorem, hence, this eigenvector is the tangent vector to the equilibrium curve implicitly defined by (C.1).

(iii) As one of the eigenvalues of $\mathbf{C}(\cdot) \frac{\partial \mathbf{g}(\cdot)}{\partial \boldsymbol{m}_{t-1}}$ is zero, the other eigenvalue must be equal to its trace. It follows that the second eigenvalue of $\left[\frac{\partial m_{k, t}}{\partial m_{l, t-1}}\right]$ is equal to $1+\operatorname{trace}\left(\mathbf{C}(\cdot) \frac{\partial \mathbf{g}(\cdot)}{\partial \boldsymbol{m}_{t-1}}\right)$.

This completes the proof of the Claim.

The meaning of the unitary eigenvalue of part $(i)$ of last Claim, and that of the associated eigenvector of part (ii), is related to the very existence of a continuum of equilibria: any local displacement of variables from an equilibrium along this continuum (locally, along the eigenvector) causes neither divergence from the new position, nor convergence to the previous one (due to the unitary eigenvalue).

We pass finally to the analysis of the last eigenvalue of $J_{F, t}^{*}$, call it $\theta$.

Claim C.3. (a) $\theta$ is lower than $1 ;(b) \theta$ can be lower than -1 .

Proof. Part (a).

Owing to part (iii) of Claim C.2, this means proving that $\operatorname{trace}\left(\mathbf{C}(\cdot) \frac{\partial \mathbf{g}(\cdot)}{\partial \boldsymbol{m}_{t-1}}\right)<0$.

We rewrite $\mathbf{C}(\cdot)$ and $\frac{\partial \mathbf{g}(\cdot)}{\partial \boldsymbol{m}_{t-1}}$ starting from the definitions given in Appendix A, omitting asterisks, arguments and time subscripts for simplicity. Recall that we are evaluating jacobian matrices in a conjectural equilibrium at time $t$ (the system variables have been in that equilibrium from date 1 to date $t$ ).

As regards matrix $\mathbf{C}$, one checks that it is equal to $\left[\begin{array}{cc}\gamma_{1}+t & -t p \\ -t p & \gamma_{2}+t p^{2}\end{array}\right]^{-1}\left[\begin{array}{ll}1 & 0 \\ 0 & p\end{array}\right]$ : simply recurse (A.1) back to date 0 with constant price $p$, use definitions after (A.2), and recall the definition of $\gamma_{1}$ and $\gamma_{2}$ from section 2.3. We can thus write

$$
\mathbf{C}=\left[\left[\begin{array}{ll}
1 & 0 \\
0 & p
\end{array}\right]^{-1}\left[\begin{array}{cc}
\gamma_{1}+t & -t p \\
-t p & \gamma_{2}+t p^{2}
\end{array}\right]\right]^{-1}=\left[\begin{array}{cc}
\gamma_{1}+t & -t p \\
-t & \frac{\gamma_{2}}{p}+t p
\end{array}\right]^{-1}
$$

and hence, from the formula of the inverse of a 2 by 2 matrix,

$$
\mathbf{C}=\frac{1}{\left(\gamma_{1}+t\right)\left(\frac{\gamma_{2}}{p}+t p\right)-t^{2} p}\left[\begin{array}{cc}
\frac{\gamma_{2}}{p}+t p & t p \\
t & \gamma_{1}+t
\end{array}\right]
$$


Call $d$ the fraction in last line: one easily checks that $d=\frac{p}{\gamma_{1} \gamma_{2}+t\left(\gamma_{2}+\gamma_{1} p^{2}\right)}>0$. Consider $\frac{\partial \mathbf{g}(\cdot)}{\partial \boldsymbol{m}_{t-1}}$ : it can be written as

$$
\frac{\partial \mathbf{g}(\cdot)}{\partial \boldsymbol{m}_{t-1}}=\left[\begin{array}{cc}
\partial g / \partial \alpha & \partial g / \partial \beta \\
-\partial g / \partial \alpha & -\partial g / \partial \beta
\end{array}\right]
$$

$g(\cdot)$ was defined in (C.1) and the signs derive from the comments thereafter.

Now, go back to trace $\left(\mathbf{C}(\cdot) \frac{\partial \mathbf{g}(\cdot)}{\partial \boldsymbol{m}_{t-1}}\right)$. Considering (C.2), (C.3) and the definition of $d$, with some passages we get

$$
\operatorname{trace}\left(\mathbf{C}(\cdot) \frac{\partial \mathbf{g}(\cdot)}{\partial \boldsymbol{m}_{t-1}}\right)=d \cdot\left(\frac{\gamma_{2}}{p} \frac{\partial g}{\partial \alpha}-\gamma_{1} \frac{\partial g}{\partial \beta}\right)
$$

As regards the derivatives in (C.4), recalling that (C.1) implies $M K p^{1 /(1-\delta)}=$ $(\alpha-c \beta) / 2$ and that $p=(\alpha+c \beta) / 2 \beta$, after routine calculation one arrives at

$$
\frac{\partial g}{\partial \alpha}=-\frac{1}{2}\left(1+\frac{1}{1-\delta} \frac{\alpha-c \beta}{\alpha+c \beta}\right)<0 \text { and } \frac{\partial g}{\partial \beta}=\frac{1}{2}\left(c+\frac{1}{1-\delta} \frac{\alpha-c \beta}{\alpha+c \beta} \frac{\alpha}{\beta}\right)>0
$$

The signs depend on the observation in footnote 17 of section 2.3. Observe in passing that they justify the argument of footnote 32 of section 4. Substituting such signs in (C.4) proves part (a) of the Claim.

Part (b).

Looking at the terms appearing in (C.5), one recognizes that $\frac{1}{1-\delta}$ is the elasticity of true demand, call it $\varepsilon_{T}$, while $\frac{\alpha-c \beta}{\alpha+c \beta}$ is the inverse of the elasticity of conjectured demand at equilibrium, call it $\varepsilon_{C}$. We introduce now the following definitions: $s \equiv \frac{\epsilon_{T}}{\epsilon_{C}}$ and $\nu \equiv \frac{\gamma_{1}}{\gamma_{2}}$.

Substitute (C.5) in (C.4); using the last definitions, some passages (in particular, note that one can write $c=2 p-\alpha / \beta)$ lead to the following expression

$$
\operatorname{trace}\left(\mathbf{C}(\cdot) \frac{\partial \mathbf{g}(\cdot)}{\partial \boldsymbol{m}_{t-1}}\right)=-\frac{1}{2} \frac{1+2 \nu p^{2}+s+\nu p \frac{\alpha}{\beta}(s-1)}{\gamma_{1}+t\left(1+\nu p^{2}\right)}
$$

It is clear that for low values of $\gamma_{1}$ and $t$, and for high values of $s$ and $\nu$, (C.6) can well be lower than -2 .

Part $(b)$ of the Claim is thus proved.

As a limiting case, consider a very low value of $\gamma_{1}$, and $t=1$ : then in the fraction appearing in (C.6) the numerator exceeds the denominator by an amount which is approximately equal to $\nu p^{2}+s+\nu p(\alpha / \beta)(s-1)$, and their ratio can well be grater than 4 for high values of $s$ and $\nu$.

Proof. The proof of Proposition 2 is obvious, collecting Claims (C.1)-(C.3). In particular, a high value of $s$ means that the true elasticity $\varepsilon_{T}$ is greater than the 
conjectured one $\varepsilon_{C}$; and a high value of $\nu$ means that the the prior precision $\gamma_{2}$ of the parameter $\beta$ is lower than the prior precision $\gamma_{1}$ of the parameter $\alpha$.

Corollary C.1. A MTPE exists.

Proof. As argued in the text, the condition for maximum profits is $\frac{p-c}{p}=\frac{1}{\epsilon_{T}}$. Hence maximum true profits require $p^{* *}=\frac{c}{\delta}$, while the firm maximizes conjectured profits at $p^{*}=\frac{\alpha}{2 \beta}+\frac{c}{2}$. Equating the two terms leads to $\alpha=c\left(\frac{2-\delta}{\delta}\right) \beta$. Substituting this into (4.1), we get

$$
\left(\frac{c(2-\delta)}{2 \delta}+\frac{c}{2}\right)^{\frac{1}{\delta-1}} M K^{*}=\frac{c \beta(2-\delta)}{2 \delta}-\frac{c \delta}{2}
$$

and after some manipulation

$$
\beta=\left(\frac{c}{\delta}\right)^{\frac{2-\delta}{\delta-1}} M K^{*}>0 \quad \Longrightarrow \quad \alpha=c\left(\frac{2-\delta}{\delta}\right) \beta>0
$$

which proves existence of an economically meaningful MTPE.

Corollary C.2. The MTPE is stable.

Proof. Trivial. In such an equilibrium $\epsilon_{T}=\epsilon_{C}$, i.e. $s=1$; substituting this into (C.6) we get:

$$
\operatorname{trace}\left(\mathbf{C}(\cdot) \frac{\partial \mathbf{g}(\cdot)}{\partial \boldsymbol{m}_{t-1}}\right)=-\frac{1+\nu p^{2}}{\gamma_{1}+t\left(1+\nu p^{2}\right)}>-1
$$

and, using part (iii) of Claim C.2, $0<\theta<1$.

Corollary C.3. For small values of $t$ and $\gamma_{1}$, if $\alpha$ and $\beta$ decrease along the CEmanifold defined by (4.1), CE's become more unstable.

Proof. We need some preliminaries. As we know, by the implicit function theorem applied to (4.1), the CE-manifold is an increasing curve. Indeed, by using (C.5):

$$
\frac{\mathrm{d} \alpha}{\mathrm{d} \beta}=\frac{c+\frac{\epsilon_{T} \alpha}{\epsilon_{C} \beta}}{1+\frac{\epsilon_{T}}{\epsilon_{C}}}>0
$$

Henceforth, the total derivative symbol means movements along the CE-manifold.

First, we prove that as $\alpha$ and $\beta$ increase along along the CE-manifold, the $\frac{\alpha}{\beta}$ ratio decreases. In fact, the following implication is obvious:

$$
0>\frac{\mathrm{d} \frac{\alpha}{\beta}}{\mathrm{d} \beta}=\frac{1}{\beta} \frac{\mathrm{d} \alpha}{\mathrm{d} \beta}-\frac{\alpha}{\beta^{2}} \quad \Longleftrightarrow \quad \frac{\mathrm{d} \alpha}{\mathrm{d} \beta}<\frac{\alpha}{\beta}
$$

Using (C.7), then, the inequalities in (C.8) are equivalent to

$$
c+\frac{\epsilon_{T} \alpha}{\epsilon_{C} \beta}<\frac{\alpha}{\beta}+\frac{\epsilon_{T} \alpha}{\epsilon_{C} \beta}
$$


which in turn does hold true by the assumption $\alpha / \beta>c$ (see footnote 14).

Next, rewrite the price set by the firm, and the ratio $s$, as functions of $\frac{\alpha}{\beta}$, that is $p=\frac{\frac{\alpha}{\beta}+c}{2}$ and $s=\frac{\epsilon_{t}}{\epsilon_{C}}=\epsilon_{T} \frac{\frac{\alpha}{\beta}-c}{\frac{\alpha}{\beta}+c}$. By simple algebra one has: $\frac{\mathrm{d} p}{\mathrm{~d} \frac{\alpha}{\beta}}=\frac{1}{2}>0$ and $\frac{\mathrm{d} s}{\mathrm{~d} \frac{\alpha}{\beta}}=\epsilon_{T} \frac{2 c}{\left(\frac{\alpha}{\beta}+c\right)^{2}}>0$. We have thus shown the following properties:

$$
\frac{\mathrm{d} \frac{\alpha}{\beta}}{\mathrm{d} \beta}<0, \quad \frac{\mathrm{d} p}{\mathrm{~d} \frac{\alpha}{\beta}}>0, \quad \frac{\mathrm{d} s}{\mathrm{~d} \frac{\alpha}{\beta}}>0
$$

We pass now to the proof of the Corollary. Inspection of (C.6) reveals that for $s \leq 2 \mathrm{CE}$ 's are stable; in addition, high values of $t$ and $\gamma_{1}$ reinforce stability. Therefore, in order to find unstable CEs we must set low values of these parameters, along with $s>2$ : hence, we take $t=1$ and $\gamma_{1}=0.5$. Substituting these values in (C.6), with some manipulation we can write it as:

$$
\operatorname{trace}\left(\mathbf{C}(\cdot) \frac{\partial \mathbf{g}(\cdot)}{\partial \boldsymbol{m}_{t-1}}\right)=-1-\frac{\left(\nu p \frac{\alpha}{\beta}\right)(s-1)}{1+2\left(1+\nu p^{2}\right)}+\frac{1}{1+2\left(1+\nu p^{2}\right)}
$$

$\frac{\alpha}{\beta}$ is decreasing in $\alpha$ and $\beta$, by the first inequality in (C.9). Proving the Corollary is thus equivalent to showing that the derivative of (C.10) w.r.t. $\frac{\alpha}{\beta}$ is negative.

Using the second inequality in (C.9), we see that the last addendum of (C.10) is indeed decreasing in $\frac{\alpha}{\beta}$, so we can focus on the second addendum.

Define $f\left(\frac{\alpha}{\beta}\right) \equiv\left(\nu p \frac{\alpha}{\beta}\right)(s-1)$ and $g\left(\frac{\alpha}{\beta}\right) \equiv 1+2\left(1+\nu p^{2}\right)$. Hence, the derivative of $-\frac{\left(\nu p \frac{\alpha}{\beta}\right)(s-1)}{1+2\left(1+\nu p^{2}\right)}$ with respect to $\frac{\alpha}{\beta}$ is negative if and only if $\frac{f^{\prime}(\cdot)}{f(\cdot)}>\frac{g^{\prime}(\cdot)}{g(\cdot)}$.

By simple algebra:

$$
\frac{f^{\prime}\left(\frac{\alpha}{\beta}\right)}{f\left(\frac{\alpha}{\beta}\right)}=\frac{\frac{\mathrm{d} s}{\mathrm{~d} \frac{\alpha}{\beta}}\left(\nu p \frac{\alpha}{\beta}\right)+(s-1)\left(\nu \frac{\alpha}{2 \beta}+\nu p\right)}{\left(\nu p \frac{\alpha}{\beta}\right)(s-1)}>\frac{\nu \frac{\alpha}{2 \beta}+\nu p}{\nu p \frac{\alpha}{\beta}}=\frac{\nu\left(2 p-\frac{c}{2}\right)}{\nu p \frac{\alpha}{\beta}}
$$

The inequality comes from $\frac{\mathrm{d} s}{\mathrm{~d} \frac{\alpha}{\beta}}>0$, the third expression in (C.9), together with $s>2$; the last passage comes from the definition of the price set by the firm.

Again by simple algebra:

$$
\frac{g^{\prime}\left(\frac{\alpha}{\beta}\right)}{g\left(\frac{\alpha}{\beta}\right)}=\frac{2 \nu p}{3+2 \nu p^{2}}
$$

We have thus to check $\frac{\nu\left(2 p-\frac{c}{2}\right)}{\left(\nu p \frac{\alpha}{\beta}\right)}>\frac{2 \nu p}{3+2 \nu p^{2}}$, which after some passages becomes:

$$
4 p+4 \nu p^{3}-\frac{3 c}{2}-c \nu p^{2}>2 \nu p^{2} \frac{\alpha}{\beta}=2 \nu p\left(2 p^{2}-p c\right)
$$


34 Quality Uncertainty, Risk Aversion, and New Product Diffusion

In the last passage we used again the definition of the optimal firm's price. Hence, one requires:

$$
4 p-\frac{3 c}{2}+c \nu p^{2}>0
$$

which is clearly true, being $p>c$. 